\title{
Transperineal versus transrectal multi-parametric magnetic resonance imaging fusion targeted prostate biopsy
}

\author{
Danny Rabah, FRCSC, FACS, Waleed Al-Taweel, MD, FRCSC, Farrukh Khan, MD, Mostafa Arafa, MPH, PhD, \\ Shahbaz Mehmood, MD, Alaa Mokhtar, MD, Karim Farhat, BSc, MSc, PhD.
}

\begin{abstract}

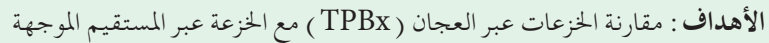

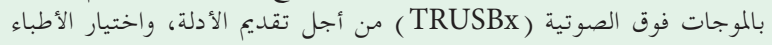
طريقة الخزعة المناسبة في ظروف مختلفة فئ فن

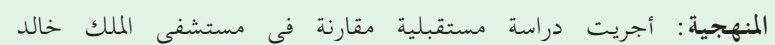

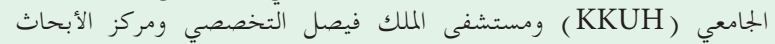

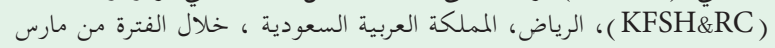

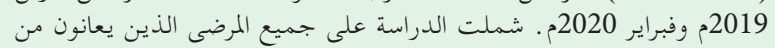

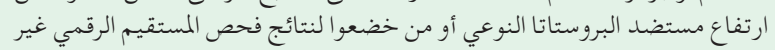

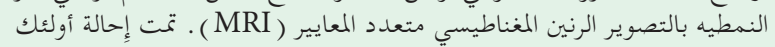

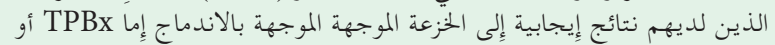

TRUSBx السرطان ووقت الإجراء.

النتائج : كان للخزعات عبر العجان و TRUSBx معدل معال مضاعفات متساوي.

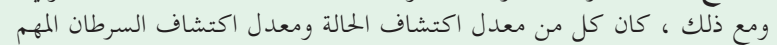

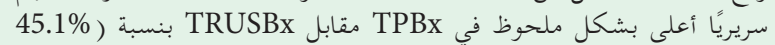

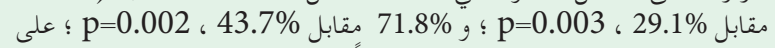

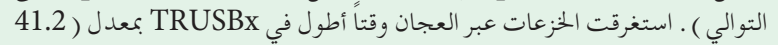

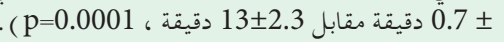

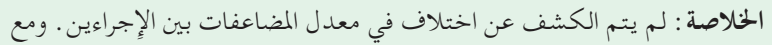

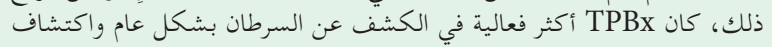

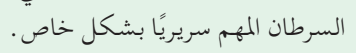

Objectives: To compare transperineal biopsies (TPBx) with transrectal ultrasound-guided biopsy (TRUSBx) in order to provide evidence, making clinicians able to select the appropriate biopsy approach under different conditions.

Methods: A comparative prospective study, conducted in King Khalid University Hospital (KKUH) and King Faisal Specialist Hospital and Research Centre (KFSH\&RC), Riyadh, Kingdom of Saudi Arabia, between March 2019 and February 2020. All patients with raised prostate-specific antigen or atypical digital rectal examination findings were subjected to multiparametric magnetic resonance imaging (MRI). Those with positive findings were referred to targeted fusionguided biopsy either TPBx or TRUSBx, randomly.
Complication rate, cancer detection rate, and procedure time were recorded.

Results: Transperineal biopsies and TRUSBx had an equivalent complication rate. However, both case detection rate and clinically significant cancer detection rate were significantly higher in TPBx versus TRUSBx $(45.1 \%$ versus $29.1 \%, p=0.003$; and $71.8 \%$ versus $43.7 \%, p=0.002$; respectively). Transperineal biopsies was a longer procedure than TRUSBx $(41.2 \pm 0.7 \mathrm{~min}$ versus $13 \pm 2.3 \mathrm{~min}, p=0.0001$ ).

Conclusion: No difference in complication rate was detected between the 2 procedures; however, TPBx was more effective for cancer detection in general and clinically significant cancer detection in particular.

Keywords: Transperineal targeted biopsy, transrectal targeted fusion biopsy, prostate cancer.

Saudi Med J 2021; Vol. 42 (6): 649-654 doi: 10.15537/smj.2021.42.6.20200771

From the Department of Surgery (Rabah, Khan), College of Medicine; from the Cancer Research Chair (Rabah, Arafa, Farhat), King Saud University, and from the Department of Urology (Rabah, Al-Taweel, Mokhtar), King Faisal Specialist Hospital \& Research Centre, Riyadh, Kingdom of Saudi Arabia.

Received 29th November 2020. Accepted 29th March 2021.

Address correspondence and reprint request to: Dr. Karim H. Farhat, Cancer Research Chair, King Saud University, Riyadh, Kingdom of Saudi Arabia.E-mail: kfarhat@ksu.edu.sa

ORCID ID: https://orcid.org/0000-0003-1871-2818

$M$ ore than 2 million cases of prostate biopsies are conducted every year all over the world, for histological confirmation of cancer. To enhance the potential for detection and localization of prostate cancer, multi-parametric magnetic resonance imaging (mpMRI) is used before biopsies are taken. ${ }^{1}$ 
The anatomical site of the prostate makes the transrectal ultrasound-guided biopsy (TRUSBx) the most suitable and convenient approach and course for biopsy, which can be accomplished in the clinic in just 10 minutes using standard TRUSBx. ${ }^{2}$ Transrectal ultrasound-guided biopsy has been the gold standard since the 1980s, although it has certain serious disadvantages. In passing through the rectal wall, the needle will contaminate the prostate with bacterial flora from the rectum. Such a risk can be minimized by targeted biopsies; however, in saturation biopsies, the needle may be required to pass more than 20 times. Each time the needle passes, it infects the prostate and the blood with rectal bacteria. Hence, the use of prophylactic antibiotics is indispensable. ${ }^{1,3}$ As an alternative to TRUSBx, transperineal biopsies (TPBx) have gained popularity as they avoid such complications. Transperineal biopsies is conducted under regional or local anesthesia and the needle passes through the disinfected perineal skin. ${ }^{4,5}$ According to the literature, magnetic resonance imaging/ultrasound (MRI/US) fusion-guided biopsy has elevated precision for cancer diagnosis. However, the best approach (transrectal or transperineal) needs further study to be standardized.

The current study aimed to provide evidence for physicians to select the proper prostate biopsy technique. We compared mpMRI/US fusion-guided techniques using the transrectal or the transperineal prostate biopsy in the setting of the first biopsy in terms of complication rate, cancer detection rate, and procedure time.

Methods. This was a comparative prospective study, conducted in 2 centers: King Khalid University Hospital and King Faisal Specialist Hospital and Research Centre, Riyadh, Kingdom of Saudi Arabia, during the period March 2019 to February 2020.

Over the period of study, all consecutive patients met the inclusion criteria and below the age of 80 years with elevated prostate-specific antigen (PSA) more than $3.5 \mathrm{ng} / \mathrm{ml}$ or abnormal findings in a digital rectal examination (DRE) such as hard mass or nodule, induration or asymmetry prostatic lobe were subjected to mpMRI of the prostate. Those with a positive

Disclosure. This study was funded by the Deanship of Scientific Research, King Saud University through the Vice Deanship of Scientific Research Chairs, Riyadh, Kingdom of Saudi Arabia. finding of a prostate imaging reporting and data system (PI-RADS) score of $\geq 3$ in the MRI were referred for targeted fusion-guided biopsy either TPBx or TRUSBx randomly, using simple random method. Patients with severe comorbidities such as heart failure and hepatic cell failure are excluded from the study.

The techniques were compared according to the complication rate (infection, hematuria, and urinary retention), length of the procedure, positive cancer detection rate (CDR), and clinically significant cancer detection rate (CSCDR). Two weeks follow up was a routine for these patients to check the final diagnosis. At that time, any possible adverse events were noted as well as how they were managed.

Cancer detection rate, CSCDR, and complication rates were the principal endpoints. For comparison bases of the risk stratification schemes (namely, Gleason score $\leq 6,7$ or $\geq 8$ ) of the European Association of Urology recommendations on prostate cancer, the CDR was stratified by Gleason score. ${ }^{6}$ The detection rate of very low-risk (VLR) prostate cancer (defined as Gleason score $=6,2$ positive biopsies, PSA density $<0.15, \leq 50 \%$ involvement on any core, and 12 or fewer sampled cores) also was investigated. Using standard terminology guidelines for adverse events version 4.0 as a guide, complications were evaluated. ${ }^{7}$

All patients underwent pre-biopsy mpMRI of their prostate. Magnetic resonance imaging images were reviewed by a dedicated radiologist and reported with PI-RADS version 2. These mpMRI images were stored in the hospital computer ICIS network and imported for fusion with real-time TRUS via the localized network system.

The transperineal MRI/US fusion-guided biopsy was carried out with the BioJet fusion system and software (D\&K Technologies, Barum, Germany). A minimum of one and preferably 2-4 cores were taken from each target lesion. All patients received only a single dose of intravenous antibiotics at the time of induction of general anesthesia and the procedure was performed in the dorsal lithotomy position.

The transrectal MRI/US fusion-guided biopsy was performed under local anesthesia with the Artemis/ Profuse system and software (Eigen, CA, USA). A systematic 12-core biopsy was performed in every patient after a minimum of $2-4$ cores were obtained from each targeted lesion, depending upon the size of a lesion. A 12 cores technique from areas of the gland using a scheme one biopsy each from both lobes in a systematic pattern of one from each medial and lateral apex, one from each lateral peripheral and medial zone and one from each medial and lateral base zone. 
Chi-square test and student's t-test were used to detect the difference between the 2 groups of all parameters for nominal and continuous variables. The significance level was set at $p \leq 0.05$. Data was analyzed using the Statistical Package for Social Sciences, version 23 (IBM Corp., Armonk, NY, USA).

Ethics approval was obtained from the Institutional Review Board at King Saud University, Riyadh Saudi Arabia (Approval No: KSU-E-18-3541). All individual participants were consented for participating in the study.

Results. Total number of patients included in the final analysis was 307 patients. Of these, 165 patients were subjected to TRUSBx and 142 patients were subjected to TPBx. The clinical characteristics of both groups are illustrated in Table 1 . Both groups are comparable, and no significant difference was detected between them for any of the measured parameters.

Table 2 compares the complication rates between TRUSBx and TPBx. Sepsis was not encountered. Urinary retention was the most common complication detected in both groups, followed by hematuria, without any significant difference between the groups. Only 6 cases $(3.6 \%)$ complained of rectal bleeding after transrectal biopsy, lasted for 24-48 hours and did not need any intervention or hospitalization.

The TPBx method showed a significantly higher detection rate of prostate cancer cases compared to TRUSBx (45.1\% vs. 29.1\%, $p=0.003)$. Clinically significant prostate cancer was distinguished in 46 cases $(71.8 \%)$ by TPBx, significantly higher than the percentage detected by TRUSBx $(43.7 \% ; p=0.002)$. The operation duration of the TPBx $(41.2 \pm 0.7$ minutes $)$ was significantly longer than the TRUSBx $(13 \pm 2.3$ minutes; $p=0.0001$ ), (Table 3 ). Out of the total cores for TPBx (1723 systematic and 338 targeted biopsy), the percentage of positive cores $(6.7 \%$ and $43.5 \%)$ was significantly higher $(p=0.0002)$ than that reported for TRUSBx (3.8\% for systematic and $21.1 \%$ for targeted biopsy) (Table 4).

Discussion. For 3 decades, urologists have primarily relied on the transrectal approach for the diagnosis of prostate cancer. There are many disadvantages to that technique, including suboptimal diagnostic accuracy and numerous adverse outcomes and complications. A plethora of research suggests that TPBx offers equivalent prostate CDR, lower infectious complications, and increased technical feasibility. The transperineal prostate biopsy is gradually earning traction around the world, given its ability to address such matters.

Our study revealed no significant difference between the 2 procedures in terms of complications; however, prostate CDR in general, and CSCDR, in particular, were significantly higher in TPBx. As the sextant transrectal biopsy protocol seemed to be insufficient for the detection of cancer prostate, studies have obtained more cores aiming at achieving higher CDR. ${ }^{8}$ Against this background, subsequent studies reported that TPBx and TRUSBx were comparable in terms of CDR. A meta-analysis by Shen et al, ${ }^{9}$ found that TPBx was not significantly different from TRUSBx with regards to CDR in general and subgroup assessment. Also, with saturation biopsy, data reinforced that TPBx and TRUSBx were effectively comparable for CDR $(31.4 \%$ vs. $25.7 \%) .{ }^{10}$ The results of Tewes et al, ${ }^{11}$ study revealed a detection rates of PCa were 39\% for TR biopsy and $75 \%$ for TP biopsy. Guo et al, ${ }^{12}$ registered a similar ability of the 2 cancer detection techniques, where the

Table 1 - Distribution of different parameters across both biopsy strategies.

\begin{tabular}{lccc}
\hline Parameters & $\begin{array}{c}\text { TRUSBx } \\
(\mathbf{n}=165)\end{array}$ & $\begin{array}{c}\text { TPBx } \\
(\mathbf{n}=142)\end{array}$ & $P$-value \\
\hline Age (mean \pm SD) & $51-80$ years $(65.1 \pm 7.8)$ & $47-80$ years $(65 \pm 8.5)$ & 0.8 \\
Prostate-specific antigen & $3.5-26 \mathrm{ng} / \mathrm{ml}(14.2 \pm 5)$ & $4-222 \mathrm{ng} / \mathrm{ml}(13.7 \pm 25.9)$ & 0.4 \\
Prostate volume & $31-170 \mathrm{cc}(61.9 \pm 34.9)$ & $26-196 \mathrm{cc}(63 \pm 22)$ & 0.7 \\
Obesity (BMI>30) (\%) & $25(15.15)$ & $24(16.9)$ & 0.6 \\
Prior prostate biopsy with negative finding (\%) & $23(14)$ & $12(8.4)$ & 0.6 \\
Present positive DRE (\%) & $15(9)$ & $14(9.8)$ & 0.8 \\
Number of lesions & & & 0.7 \\
$\quad$ PI-RADS 3 & 35 & 30 & \\
$\quad$ PI-RADS 4 & 19 & 25 & \\
$\quad$ PI-RADS 5 & 16 & 20 & \\
\hline
\end{tabular}

BMI: body mass index, DRE: digital rectal examination, PI-RADS: prostate imaging reporting and data system, TRUSBx - transrectal ultrasound-guided biopsy, TPBx: transperineal biopsies 
Table 2 - Comparison of TRUSBx and TPBx regarding procedure's complications.

\begin{tabular}{lccc}
\hline Complications & TRUSBx $(\mathbf{n}=165)$ & TPBx $(\mathbf{n}=142)$ & $P$-value \\
\hline Urinary retention & $7(4.2)$ & $8(5.6)$ & 0.56 \\
Urinary tract infection & 0 & 0 & - \\
Hematuria & $2(1.2)$ & $1(0.7)$ & 0.6 \\
Rectal bleeding & $6(3.6)$ & 0 & - \\
\hline
\end{tabular}

Values are presented as number and percentage (\%). TRUSBx: transrectal ultrasound-guided biopsy, TPBx - transperineal biopsies

Table 3 - Comparison of TRUSBx and TPBx regarding procedure's cancer detection rate, and duration of the procedure.

\begin{tabular}{lccc}
\hline Parameters & TRUSBx $(\mathbf{n}=165)$ & TPBx $(\mathbf{n}=142)$ & $P$-value \\
\hline $\begin{array}{l}\text { Cancer detection } \\
\text { rate }\end{array}$ & $48(29.1)$ & $64(45.1)$ & 0.003 \\
$\begin{array}{l}\text { Clinically significant cancer detection rate } \\
\quad \text { PI-RADS 3 }\end{array}$ & $2(9.5)$ & $1(2.1 \%)$ & 0.05 \\
$\quad 7(33.3)$ & $20(43.4 .1 \%)$ & \\
$\quad$ PI-RADS 4 & $12(57.1)$ & $25(54.3 \%)$ & \\
$\quad$ PI-RADS 5 & $21(43.7)$ & $46(71.8 \%)$ & 0.002 \\
$\quad \begin{array}{l}\text { Total } \\
\text { Operation duration } \\
\text { (mean } \pm \text { SD) }\end{array}$ & $13 \pm 2.3$ & $41.2 \pm 0.7$ & 0.0001 \\
\hline
\end{tabular}

Values are presented as number and percentage (\%). TRUSBx: transrectal ultrasound-guided biopsy, TPBx: transperineal biopsies, SD - standard deviation.

CDR was $35.3 \%$ vs. $31.9 \%(p=0.566)$ and the positive rate of cores was $13.9 \%$ vs. $12.5 \%(p=0.224)$ for TPBx vs. TRUSBx. In the proportion of each pathological pattern, no substantial difference was observed $(p>0.05)$. In addition, there was no significant difference between the 3 levels of CDR when it was stratified by Gleason score $(p>0.05)$. In the same context, the meta-analysis conducted by Xu et $\mathrm{al}^{13}$ and Xiang et al, ${ }^{14}$ reported that no significant differences in prostate CDR between the TPBx and TRUSBx approaches; both approaches had the same diagnostic accuracy for prostate cancer; yet, transperineal technique is safer and poses a meaningfully lower risk of infection and rectal bleeding.

Studies have shown outstanding detection rates of significant prostate cancer for anterior tumors using MRI-guided targeted or systematic TPBx. ${ }^{15}$ In contrast to the TRUSBx, a recent study found that TPBx was primarily superior in the diagnosis of prostate cancer in the apex ( $47 \%$ vs. $31 \%, p=0.043)$ and anterior lobe $(54 \%$ vs. $31 \%, p=0.04)$, given the fact that all biopsies were MRI/US fusion-guided biopsies. ${ }^{16}$ Jiang et $\mathrm{al},{ }^{17}$ reported the detection of a higher proportion
Table 4 - Comparison of positive cores between TRUSBx \& TPBx.

\begin{tabular}{lcc}
\hline Positive cores & TRUSBx & TPBx \\
\hline Systematic biopsy & $76(3.8)$ & $115(6.7)$ \\
Target biopsy & $85(21.1)$ & $147(43.5)$ \\
\hline
\end{tabular}

Values are presented as number and percentage (\%). TRUSBx: transrectal ultrasound-guided biopsy, TPBx: transperineal biopsies

of clinically significant prostate cancer using TPBx procedures. Transperineal biopsies approach permits the operator to easily and better reach the anterior zone of the gland. Because patients are asleep under a general anesthetic for transperineal biopsy, discomfort during the biopsy process is not an issue, allowing many more samples to be taken and so increasing the detection rate. ${ }^{18-20}$

The rate of complications in the current study was comparable between the 2 procedures, with the most common being urinary retention, and no cases of sepsis. A study by Young et al, ${ }^{21}$ demonstrated a comparable complication rate between TRUSBx and TPBx. The greatest complication was acute urinary retention, which occurred in 28 cases following TPBx (6.71\%). $\mathrm{Xu}$ et $\mathrm{al},{ }^{13}$ reported from their meta-analysis that no significant difference was found in abnormal DRE findings, prostate volume, Gleason score or serum PSA level measurement between the 2 procedures. This meta-analysis also found no significant variations in the related complications between these 2 techniques. ${ }^{13}$

In a study presented at the American Urological Association's ${ }^{16}$ in 2019 annual meeting that focused on differences in cancer detection between the 2 procedures, TPBx was found to be associated with meaningfully lesser infectious complications, though with an augmented risk of urinary retention.

Several studies have examined factors associated with an augmented risk of developing sepsis following prostate biopsy. There is evidence to recommend that factors such as prostatic enlargement, preceding exposure to antibiotic, previous hospitalization or surgery, prior TRUSBx, history of diabetes mellitus, immune deficiency, recurrent urinary tract infections, and overweight or obesity are associated with an enlarged risk of developing infection as a complication of prostate biopsy. ${ }^{22-25}$ The available data for the current study (prostatic enlargement, previous hospitalization or surgery and recurrent urinary tract infections) did 
not have a significant influence on the postoperative complication. There are worries about probable problems leading to a sluggish acceptance rate of TPBx. These worries appear to be centered on the observed necessity for general anesthesia, longer duration, and higher charge. The mean operating time of TPBx was significantly longer $(41 \pm 0.7 \mathrm{~min})$ in comparison to TRUSBx $(13 \pm 2.3 \mathrm{~min})$ in the current study, which was consistent with that of Guo et $\mathrm{al}^{12}$ and Kravchick et $\mathrm{al},{ }^{26}$ who reported an average of only 8.45 minutes for each TRUSBx procedure. This is related to the use requirement of operating room setup, anesthesia, and patient positioning.

Study limitations. Firstly, the sample size is small, but as there is a very low prevalence of prostate cancer in our region, all cases admitted to the hospital that met the inclusion criteria were included in the study. Secondly, pain was not assessed. Finally, as the study did not include a direct control group, it cannot be conclusively indicated that the decreased complication rates of infections, hematuria, and urinary retention in this study were directly attributable to one factor in specific. It may be valuable for future studies to include a matched comparison group to more clearly establish which factors decrease complications.

In conclusion, our study suggests comparable rates of complications between TRUSBx and TPBx. In contrast, TPBx was more effective, with the prostate CDR in general, and CSCDR in particular, significantly higher in TPBx compared with TRUSBx. The clinical characteristics of the patient had no impact on optimizing prostate biopsy in this study. No significant difference was detected between the complication rates of the 2 procedures; however, TPBx was more effective for prostate cancer detection in general and clinically significant cancer detection in particular.

Acknowledgment. The authors are grateful to the Deanship of Scientific Research, King Saud University for funding through the Vice Deanship of Scientific Research Chairs Riyadh, Kingdom of Saudi Arabia.

\section{References}

1. Borghesi M, Ahmed H, Nam R, Schaeffer E, Schiavina R, Taneja S, et al. Complications after systematic, random, and image-guided prostate biopsy. Eur Urol 2017; 71: 353-365.

2. Scherr DS, Eastham J, Ohori M, Scardino PT. Prostate biopsy techniques and indications: when, where, and how? Semin Urol Oncol 2002; 20: 18-31.

3. Walker JT, Singla N, Roehrborn CG. Reducing infectious complications following transrectal ultrasound-guided prostate biopsy: a systematic review. Rev Urol 2016; 18: 73-89.
4. Davis P, Paul E, Grummet J. Current practice of prostate biopsy in Australia and New Zealand: a survey. Urol Ann 2015; 7 : 315-319.

5. Murphy DG, Grummet JP. Planning for the post-antibiotic era - why we must avoid TRUS-guided biopsy sampling. Nat Rev Urol 2016; 13: 559-560.

6. Heidenreich A, Bastian PJ, Bellmunt J, Bolla M, Joniau S, van der Kwast T, et al. EAU guidelines on prostate cancer. part 1: screening, diagnosis, and local treatment with curative intentupdate 2013. Eur Urol 2014; 65: 124-137.

7. National Institutes of Health. Common terminology criteria for adverse events v4.0. Rockville (MD): Capital Technology Information Services, Inc; 2020.

8. Eskew LA, Bare RL, McCullough DL. Systematic 5 region prostate biopsy is superior to sextant method for diagnosing carcinoma of the prostate. J Urol 1997; 157: 199-202.

9. Shen PF, Zhu YC, Wei WR, Li YZ, Yang J, Li YT, et al. The results of transperineal versus transrectal prostate biopsy: a systematic review and meta-analysis. Asian J Androl 2012; 14 : 310-315.

10. Abdollah F, Novara G, Briganti A, Scattoni V, Raber M, Roscigno $\mathrm{M}$, et al. Trans-rectal versus trans-perineal saturation rebiopsy of the prostate: is there a difference in cancer detection rate? Urology 2011; 77: 921-925.

11. Tewes S, Peters I, Tiemeyer A, Peperhove M, Hartung D, Pertschy S, et al. Evaluation of MRI/ultrasound fusion-guided prostate biopsy using transrectal and transperineal approaches. Biomed Res Int 2017; 2017: 2176471.

12. Guo LH, Wu R, Xu HX, Xu JM, Wu J, Wang S, et al. Comparison between ultrasound guided transperineal and transrectal prostate biopsy: a prospective, randomized, and controlled trial. Sci Rep 2015; 5: 16089.

13. Xue J, Qin Z, Cai H, Zhang C, Li X, Xu W, et al. Comparison between transrectal and transperineal prostate biopsy for detection of prostate cancer: a meta-analysis and trial sequential analysis. Oncotarget 2017; 8: 23322-23336.

14. Xiang J, Yan H, Li J, Wang X, Chen H, Zheng X. Transperineal versus transrectal prostate biopsy in the diagnosis of prostate cancer: a systematic review and meta-analysis. World J Surg Oncol 2019; 17: 31 .

15. Grummet J, Pepdjonovic L, Huang S, Anderson E, Hadaschik B. Transperineal vs. transrectal biopsy in MRI targeting. Transl Androl Urol 2017; 6: 368-375.

16. American Urological Association. Transperineal vs transrectal MRI/US fusion for prostate cancer detection. Chicago (IL):American Urological Association; 2019.

17. Jiang CY, Shen PF, Wang C, Gui HJ, Ruan Y, Zeng H, et al. Comparison of diagnostic efficacy between transrectal and transperineal prostate biopsy: a propensity score-matched study. Asian J Androl 2019; 21: 612-617.

18. de Gorski A, Rouprêt M, Peyronnet B, Le Cossec C, Granger B, Comperat E, et al. Accuracy of magnetic resonance imaging/ ultrasound fusion targeted biopsies to diagnose clinically significant prostate cancer in enlarged compared to smaller prostates. J Urol 2015; 194: 669-673.

19. Barqawi AB, Krughoff KJ, Eid K. Current challenges in prostate cancer management and the rationale behind targeted focal therapy. Adv Urol 2012; 2012: 862639.

20. Tu X, Liu Z, Chang T, Qiu S, Xu H, Bao Y, et al. Transperineal magnetic resonance imaging-targeted biopsy may perform better than transrectal route in the detection of clinically significant prostate cancer: systematic review and meta-analysis. Clin Genitourin Cancer 2019; 17: e860-e870. 
21. Young R, Norris B, Reeves F, Peters JS. A retrospective comparison of transrectal and transperineal prostate biopsies: experience of a single surgeon. J Endourol 2019; 33: 498-502.

22. Loeb S, van den Heuvel S, Zhu X, Bangma CH, Schröder FH, Roobol MJ. Infectious complications and hospital admissions after prostate biopsy in a European randomized trial. Eur Urol 2012; 61: 1110-1114.

23. Steensels D, Slabbaert K, De Wever L, Vermeersch P, Van Poppel $\mathrm{H}$, Verhaegen J. Fluoroquinolone-resistant E. coli in intestinal flora of patients undergoing transrectal ultrasound-guided prostate biopsy--should we reassess our practices for antibiotic prophylaxis? Clin Microbiol Infect 2012; 18: 575-581.
24. Cussans A, Somani BK, Basarab A, Dudderidge TJ. The role of targeted prophylactic antimicrobial therapy before transrectal ultrasonography-guided prostate biopsy in reducing infection rates: a systematic review. BJU Int 2016; 117: 725-731.

25. Halpern JA, Sedrakyan A, Dinerman B, Hsu WC, Mao J, $\mathrm{Hu}$ JC. Indications, utilization and complications following prostate biopsy: New York State analysis. J Urol 2017; 197: 1020-1025.

26. Kravchick S, Peled R, Ben-Dor D, Dorfman D, Kesari D, Cytron S. Comparison of different local anesthesia techniques during TRUS-guided biopsies: a prospective pilot study. Urology 2005; 65: 109-113. 